\title{
Aplicativo de telemedicina como plataforma de comunicação interprofissional entre a atenção primária e a especializada
}

\author{
Telemedicine application as an interprofessional communication platform between primary and \\ specialized care \\ Aplicación de la telemedicina como plataforma de comunicación interprofesional entre atención \\ primaria y especializada
}

\section{Resumo}

O compartilhamento do cuidado entre as equipes da Atenção Primária à Saúde (APS) e Atenção Especializada (AE) favorece a qualificação progressiva dos profissionais atuantes em cuidados primários, aumentando sua capacidade resolutiva e seu escopo de atuação, além de proporcionar redução de encaminhamentos para a AE. O presente estudo objetivou descrever o desenvolvimento de um aplicativo para smartphone, denominado IntegRAS, concebido para proporcionar um canal de comunicação entre profissionais generalistas atuantes na APS e especialistas atuantes na área de Endocrinologia. Secundariamente, propõe-se a realizar um teste piloto de usabilidade do IntegRAS com médicos voluntários da APS e de um hospital universitário. Após o levantamento dos requisitos funcionais e definição da arquitetura do projeto, o aplicativo foi desenvolvido utilizando tecnologia multiplataforma (Android e iOS), e disponibilizado em versão de teste a cinco profissionais médicos, sendo 2 especialistas e 3 generalistas, durante um período de 90 dias. Aos participantes foram enviados questionários de usabilidade tendo como referência a System Usability Scale (SUS). O aplicativo desenvolvido atuou como uma ferramenta de suporte à tomada de decisão clínica de médicos generalistas na APS. O questionário SUS apresentou pontuações de 87.5 a 95, atribuindo ao IntegRAS uma avaliação ótima na escala de usabilidade e respaldando-o, com sua interface e características atuais, como um recurso tecnológico eficiente na integração entre as equipes de saúde. O IntegRAS se constituiu um instrumento para auxílio na assistência à prática médica, com potencial de ser útil na educação permanente em saúde para os profissionais generalistas da APS.

Palavras-chave: Aplicativos móveis; Atenção Primária à Saúde; Atenção Secundária à Saúde.

\footnotetext{
Abstract

The sharing of care between the Primary Health Care (PHC) and Specialized Care teams favors the progressive qualification of professionals working in primary care, increasing their resolving capacity and scope of action, in addition to providing a reduction in referrals for the Specialized Care. This study aimed to describe the development of a smartphone application, called IntegRAS, designed to provide a communication channel between general practitioners working in PHC and endocrinologists. Secondarily, it is proposed to carry out a pilot usability test of
} 
IntegRAS with volunteer doctors from APS and a university hospital. After surveying the functional requirements and defining the project architecture, the application was developed using multi-platform technology (Android and iOS) and made available in a trial version to five medical professionals, 2 specialists and 3 generalists, for a period of 90 days. Usability questionnaires were sent to the participants with reference to the System Usability Scale (SUS). The developed application acted as a tool to support clinical decision making for general practitioners in PHC. The SUS questionnaire presented scores from 87.5 to 95, giving IntegRAS an excellent rating on the usability scale and supporting it, with its interface and current characteristics, as an efficient technological resource in the integration between health teams. The IntegRAS was an instrument to aid in the assistance to medical practice, with the potential to be useful in continuing health education for general practitioners of PHC.

Keywords: Mobile applications; Primary Health Care; Secondary Care.

\section{Resumen}

El reparto asistencial entre los equipos de Atención Primaria de Salud (APS) y Atención Especializada (AE) favorece la progresiva cualificación de los profesionales que laboran en atención primaria, aumentando su capacidad resolutiva y ámbito de actuación, además de proporcionar una reducción de las derivaciones para la AE. Este estudio tuvo como objetivo describir el desarrollo de una aplicación para teléfonos inteligentes, denominada IntegRAS, diseñada para proporcionar un canal de comunicación entre los médicos generales que trabajan en la APS y los especialistas que trabajan en el campo de la endocrinología. En segundo lugar, se propone realizar una prueba piloto de usabilidad de IntegRAS con médicos voluntarios de APS y un hospital universitario. Luego de relevar los requerimientos funcionales y definir la arquitectura del proyecto, la aplicación fue desarrollada utilizando tecnología multiplataforma (Android e iOS), y se puso a disposición en una versión de prueba para cinco profesionales médicos, 2 especialistas y 3 generalistas, por un período de 90 días. Se enviaron cuestionarios de usabilidad a los participantes con referencia al System Usability Scale (SUS). La aplicación desarrollada actuó como una herramienta para apoyar la toma de decisiones clínicas para los médicos generales en la APS. El cuestionario SUS presentó puntajes de 87.5 a 95, otorgando a IntegRAS una calificación excelente en la escala de usabilidad y apoyándolo, con su interfaz y características actuales, como un recurso tecnológico eficiente en la integración entre equipos de salud. El IntegRAS fue un instrumento de ayuda en la asistencia a la práctica médica, con el potencial de ser útil en la educación continua para la salud de los médicos generales en APS.

Palabras clave: Aplicaciones móviles; Atención Primaria de Salud; Atención Secundaria de Salud.

\section{Introdução}

A problemática da relação entre a Atenção Primária à Saúde (APS) e a Atenção Especializada (AE) como partes de um sistema de saúde fragmentado, com profissionais atuando de forma isolada e independente, vem sendo objeto de discussões ao longo dos últimos anos no Brasil (Mendes, 2011). Inserir os níveis de atenção à saúde em um contexto de rede, tendo a APS como porta de entrada e ordenadora dos fluxos, é a lógica da chamada Rede de Atenção à Saúde (RAS), que pressupõe uma comunicação orgânica entre seus componentes, indo além do sistema clássico de referência e contrarreferência de casos, no sentido da construção de relacionamentos interpessoais, apoio matricial e compartilhamento do cuidado entre as equipes assistenciais (Brasil, 2019; Gonçalves et al., 2017; Mendes, 2011; Peiter et al., 2019).

A partilha dos planos de cuidado dos usuários entre APS e AE é capaz de substituir encaminhamentos, além de favorecer a qualificação progressiva dos profissionais atuantes em cuidados primários, aumentando sua capacidade resolutiva e seu escopo de atuação (Brasil, 2019). A prestação remota de cuidados em saúde mediada pelo avanço das tecnologias da informação e comunicação (TIC) vem oportunizando um importante incremento na integração entre profissionais da atenção básica e especialistas, trazendo perspectivas de melhoria dos desfechos na transição de cuidados da APS para a AE (Muniz, Santos, Araújo, Oliveira, \& Simões, 2021).

O crescente desenvolvimento tecnológico vivenciado nas últimas décadas tem proporcionado a ampliação do alcance e a facilitação das práticas assistenciais em saúde. Especialmente com o advento e adesão global dos smartphones, conceituados de forma simplificada e em tradução literal como "telefones inteligentes" em referência à alta capacidade de processamento destes dispositivos (Coutinho, 2014), as TIC rapidamente adentraram no campo da saúde, tanto através de 
plataformas comunicacionais vinculadas à internet (web-based) quanto por meio de aplicativos, ou simplesmente apps - do inglês applications.

Em recente revisão integrativa acerca dos impactos de sistemas eletrônicos de comunicação no setor saúde, apenas plataformas vinculadas à web foram reportadas (Muniz et al., 2021). Buscas na literatura específicas para aplicativos revelaram diversos projetos de desenvolvimento de apps com cunho assistencial, prevendo suporte remoto para profissionais generalistas em áreas distintas, com destaque para a condução de casos de queimaduras (Blom et al, 2018; Martinez et al, 2018; Wallis et al, 2016; Wiktor et al., 2018) e para a área de dermatologia (Hasbún Zegpi, et al, 2020; Pecina, et al, 2017; Rizvi, et al, 2020; Yang, et al, 2019). Junto às lojas virtuais de aplicativos App Store e Google Play, a busca de anterioridade encontrou duas plataformas bastante semelhantes, ofertadas para discussão de casos clínicos entre profissionais de saúde de todo o mundo através do compartilhamento de fotos e troca de comentários nas imagens: o app canadense Figure 1 (https://www.figure1.com, recuperado em 16 de outubro, 2021), e o britânico MedShr (https://en.medshr.net, recuperado em 16 de outubro, 2021). Não foram identificados, no entanto, publicações ou aplicativos representando fielmente o conjunto de contexto e proposta do aplicativo objeto deste artigo.

Nesta perspectiva, o presente estudo objetiva primariamente descrever o desenvolvimento de um aplicativo para smartphone concebido para viabilizar um canal de comunicação direta entre profissionais generalistas atuantes na APS e especialistas atuantes na área de Endocrinologia, com o intuito de prover suporte à tomada de decisão clínica através do compartilhamento do cuidado. Secundariamente, realizar um teste piloto de usabilidade do aplicativo ora desenvolvido.

\section{Metodologia}

Trata-se de um estudo de intervenção, cuja execução deu-se em três etapas, sendo: 1) desenvolvimento do aplicativo; 2) teste piloto; 3) avaliação da usabilidade do aplicativo junto aos participantes do teste piloto.

O estudo foi submetido à apreciação do Comitê de Ética em Pesquisa da Universidade Federal de Sergipe (UFS), com Certificado de Apresentação de Apreciação Ética de n 29124720.1.0000.5546, tendo sido aprovado para execução sob parecer de $\mathrm{n}^{\circ}$ 3.999.730. A participação dos profissionais no teste piloto de usabilidade foi voluntária e sob consentimento informado.

\section{Desenvolvimento do aplicativo}

O desenvolvimento do aplicativo envolveu uma equipe multidisciplinar. Na condição de idealizadoras do projeto, participaram as autoras deste artigo. Da área de programação participaram outros quatro profissionais, sendo um programador front-end, um programador back-end, um designer e um profissional de suporte.

$\mathrm{O}$ aplicativo foi denominado IntegRAS, em uma referência à pretendida integração entre profissionais atuantes em uma mesma Rede de Atenção à Saúde. A especialidade de Endocrinologia foi escolhida por ser uma área eminentemente clínica, e sensível à discussão remota de casos e compartilhamento do cuidado entre profissionais da saúde.

As funcionalidades do IntegRAS, foram definidas com base nas recomendações do Protocolo de Encaminhamento da Atenção Básica para a Atenção Especializada, do Ministério da Saúde (Brasil, 2015), por meio do qual determinaram-se as principais doenças de cunho endocrinológico que são motivo de encaminhamento para os ambulatórios de atenção especializada em Endocrinologia, bem como, as informações mínimas necessárias para o profissional especialista emitir um parecer acerca de um determinado caso clínico.

Assim, foram criadas funcionalidades para debates clínicos relacionados a cinco doenças (diabetes mellitus, hipotireoidismo, hipertireoidismo, nódulos de tireoide e obesidade), bem como para outras condições de interesse por parte do solicitante. 
A arquitetura adotada para este projeto seguiu o padrão Model-View-Controller $(M V C)$, que divide as funcionalidades do software em camadas distintas. Após o levantamento dos requisitos funcionais, o processo de desenvolvimento do aplicativo seguiu com a prototipação, utilizando a ferramenta Figma, destinada à criação gráfica de esquemas de design e experiência de usuário, e na sequência, com a elaboração do back-end e criação do banco de dados utilizando o sistema gerencial PostgreSQL (versão 10.18).

O aplicativo foi desenvolvido com a framework React Native (versão 0.64), tecnologia que possibilita a criação de aplicativos móveis multiplataforma (Android e iOS) utilizando linguagem JavaScript (versão ECMAScript 2018), onde o código de programação criado é convertido para a linguagem nativa do sistema operacional a ser utilizado. Assim, a implantação deste aplicativo foi realizada utilizando o ambiente de desenvolvimento integrado Android Studio (versão 4.2) e a ferramenta Xcode (versão 12.4) para o sistema iOS. Quanto ao back-end do aplicativo, a linguagem de servidor escolhida foi o Node.js (versão LTS 12.17.0), também baseada em JavaScript.

\section{Teste piloto}

A divulgação da proposta do aplicativo para médicos generalistas foi realizada mediante contatos prévios com a Coordenação de Educação Permanente da Secretaria Municipal de Saúde de São Cristóvão/SE e a Coordenação do Internato em Saúde da Família do curso de Medicina da UFS, a fim de verificar o interesse e disponibilidade dos profissionais integrantes das equipes da ESF em participar do teste de usabilidade. Para os médicos especialistas, a apresentação do projeto ocorreu em reunião junto à equipe de Endocrinologia do Hospital Universitário da UFS. Voluntariamente, três generalistas e dois endocrinologistas se dispuseram a participar do teste piloto do aplicativo.

O protótipo do aplicativo IntegRAS foi disponibilizado durante 90 dias aos cinco participantes do estudo piloto, em versão de teste, através do aplicativo TestFlight para o sistema operacional iOS e por meio de um link para o sistema Android. Previamente ao início do teste de uso, os participantes receberam um vídeo de conteúdo instrucional acerca das configurações e funcionalidades do aplicativo.

O procedimento de teste se deu mediante a utilização do aplicativo pelos médicos generalistas, por livre demanda, para disporem suas dúvidas clínicas no contexto da especialidade de Endocrinologia. Os médicos especialistas, por sua vez, receberam uma notificação de chegada de cada interconsultoria e tiveram até $48 \mathrm{~h}$ para responder às solicitações recebidas, transferindo a tomada de decisão ao médico da APS solicitante.

\section{Avaliação da usabilidade do aplicativo}

Ao final do período de testagem do aplicativo, os participantes responderam a um questionário composto por duas seções. A primeira seção correspondeu a dez itens baseados no teste denominado System Usability Scale (SUS), cuja proposta é avaliar aspectos relativos à usabilidade do aplicativo de forma mais subjetiva, utilizando uma escala Likert de concordância de cinco pontos. Às respostas, foram atribuídos valores de um a cinco, respectivamente para "discordo fortemente", "discordo parcialmente", "nem discordo nem concordo", "concordo parcialmente" e "concordo fortemente" (Brooke, 1996). A segunda seção foi composta de sete itens para os participantes vinculados à APS e três para os endocrinologistas, sendo o primeiro item - comum aos dois grupos de participantes - um questionamento sobre se mudariam algo no aplicativo e, em caso afirmativo, o que mudariam. Os demais itens foram compostos por afirmações que utilizaram para resposta a escala Likert de concordância anteriormente mencionada.

O escore total do SUS foi calculado da seguinte maneira: a) nos itens ímpares: subtraiu-se 1 da resposta do usuário; b) nos itens pares: subtraiu-se 5 das respostas do usuário; c) as respostas foram somadas e o total foi multiplicado por 2.5, obtendo-se assim o valor total do $S U S$, cuja variação ocorre entre 0 e 100 pontos (Martins et al., 2015), onde pontuações 
abaixo de 70 significariam produtos julgados como marginais, passíveis de análise e melhorias, enquanto produtos melhores estariam entre 70 e 80 , e aqueles verdadeiramente superiores obteriam pontuações acima de 90 (Bangor et al, 2008).

\section{Resultados}

O sistema do aplicativo foi projetado para armazenar informações do usuário (médicos generalistas e especialistas) e dados do paciente em um servidor de rede na internet, receber dados (informações clínicas do paciente) e transmiti-los ao médico especialista, e finalmente, responder com suporte definido à tomada de decisão de manejo clínico pelo profissional de saúde generalista.

A Figura 1 ilustra a tela inicial do layout do médico solicitante (generalista), onde constam no dashboard os registros dos casos aguardando o parecer do especialista (FILA) e aqueles com o parecer já emitido (RETORNOS); na aba Pacientes, constam os registros de todos os casos criados e o ícone onde o usuário insere novos casos (+). Na sequência, exemplifica-se o fluxograma de cadastro de paciente pelo médico solicitante da APS.

Figura 1 - Layout do médico generalista do IntegRAS e fluxograma de cadastro de paciente.

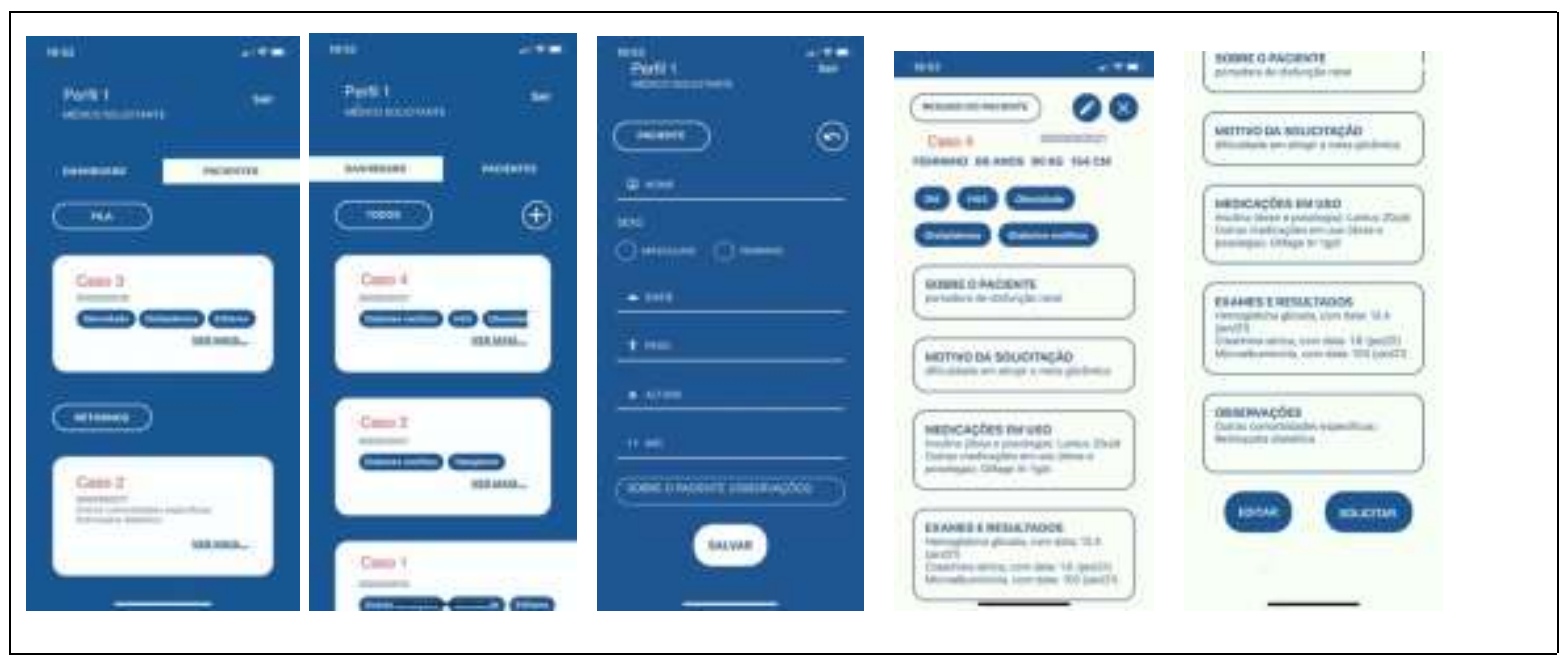

Fonte: Autores (2021).

No layout do especialista, conforme demonstrado na Figura 2, constam os campos de chegada de novos casos (SOLICITAÇÕES) e, uma vez que a interconsultoria seja respondida, o caso em questão passa a ser exibido no campo denominado RESPONDIDOS. A interação que ocorre a partir do médico especialista dá-se em formato de texto livre. 
Figura 2 - Layout do médico especialista do IntegRAS e exemplo da elaboração de resposta a uma solicitação.

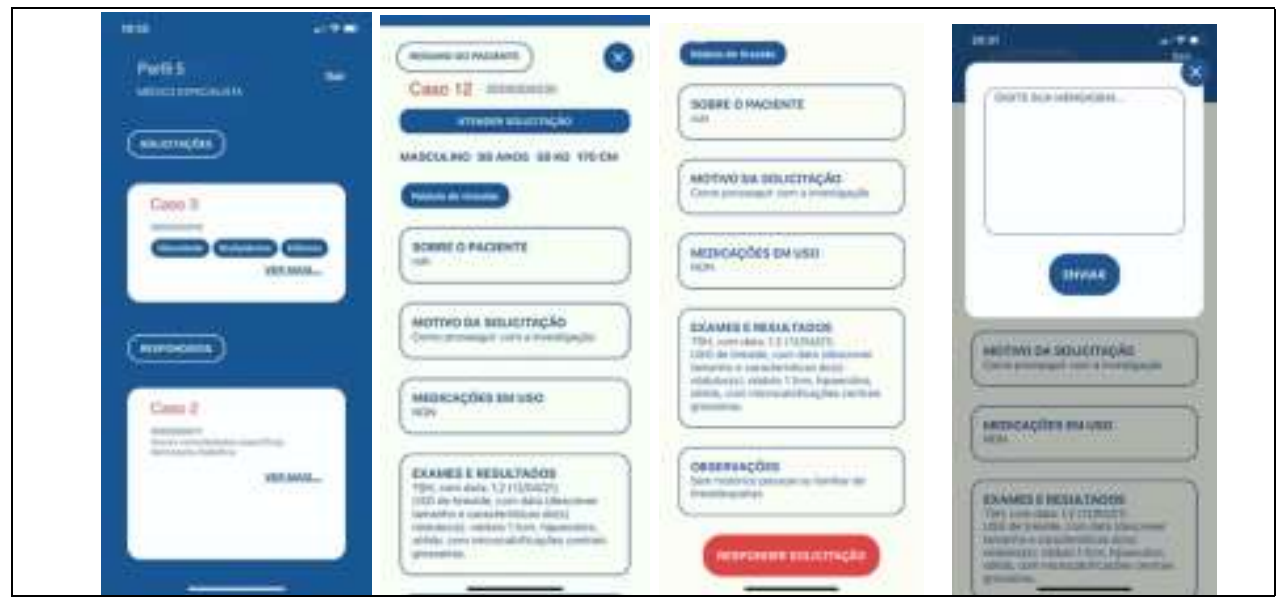

Fonte: Autores (2021).

Com relação à caracterização dos participantes do teste piloto, três médicos generalistas e dois endocrinologistas, as variáveis elencadas foram: idade, com 40\% ( $\mathrm{n}=2)$ da amostra entre 25 e 29 anos, e $60 \%(\mathrm{n}=3)$ entre 30 e 34 anos; gênero, sendo $40 \%$ (n=2) masculino e 60\% (n=3) feminino; tempo de graduação em Medicina, onde $40 \%$ (n=2) reportou formação há menos de 5 anos, $40 \%$ (n=2) entre 5 e 10 anos, e 20\% há mais de 10 anos; experiência em uso de telemedicina, foi reportada por $40 \%(n=2)$ da amostra; uso de aplicativos de apoio à decisão clínica no cotidiano de trabalho foi reportado por todos os participantes. Todos os participantes generalistas afirmaram tempo de atuação na APS menor que 5 anos. A média mensal de encaminhamentos para a especialidade de Endocrinologia foi menor que 5 pacientes, conforme respostas de dois médicos. O terceiro referiu encaminhar uma média de 5 a 10 pacientes.

Quanto à avaliação da usabilidade do aplicativo, os resultados da primeira seção do questionário (respostas obtidas para cada item e pontuações da escala SUS de cada participante) encontram-se representados respectivamente no Gráfico $1 \mathrm{e}$ na Tabela 1. Na segunda seção, os cinco participantes responderam negativamente ao questionamento inicial, sobre se mudariam algo no app. As respostas às demais perguntas do questionário pós-teste, elaboradas segundo a escala Likert de concordância, seguem representadas no Gráfico 2 . 
Gráfico 1 - Respostas ao questionário SUS aplicado aos cinco médicos participantes do teste piloto do aplicativo IntegRAS.

\section{Questionário System Usability Scale}

Item 1 Gostaria de utilizar este produto com frequência

Item 2. Considerei o produto mais complexo do que o necessário

Item 3. Achei o produto fácil de utilizar

Itern 4. Necessitaria de ajuda de um técnico para conseguir usar este produto

Item 5. Considerei que as várias funcionalidades deste produto estavam bem integradas

Item 6. Achei que este produto tinha muitas inconsistências

item 7. Suponho que a maioria das pessoas aprenderia a utilizar rapidamente este produto

Item 8 . Considerei o produto muito complicado de utilizar

Item 9. Senti-me muito confiante a utilizar este produto Item 10. Tive que aprender muito antes de conseguir lidar com este produto

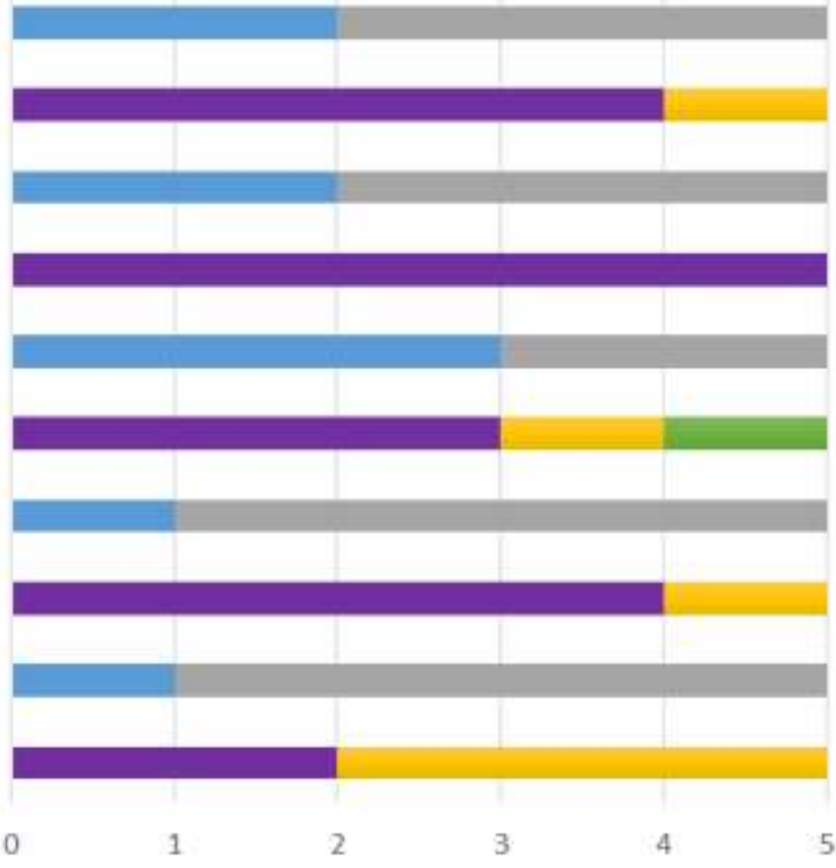

Discordo fortemente

$=$ Discordo parcialmente $\quad$ Nem discordo nem concordo

a Concordo parcialmente

= Concordo fortemente

Fonte: Autores (2021).

Tabela 1 - Pontuações obtidas no questionário SUS aplicado aos médicos participantes do teste piloto do aplicativo IntegRAS.

\begin{tabular}{llllll}
\hline Perguntas & Participante 1 & Participante 2 & Participante 3 & Participante 4 & Participante 5 \\
\hline Pergunta 1 & $5(5-1=4)$ & $5(5-1=4)$ & $4(4-1=3)$ & $4(4-1=3)$ & $5(5-1=4)$ \\
Pergunta 2 & $1(1-5=4)$ & $1(1-5=4)$ & $1(1-5=4)$ & $2(2-5=3)$ & $1(1-5=4)$ \\
Pergunta 3 & $5(5-1=4)$ & $4(4-1=3)$ & $5(5-1=4)$ & $5(5-1=4)$ & $4(4-1=3)$ \\
Pergunta 4 & $1(1-5=4)$ & $1(1-5=4)$ & $1(1-5=4)$ & $1(1-5=4)$ & $1(1-5=4)$ \\
Pergunta 5 & $5(5-1=4)$ & $4(4-1=3)$ & $4(4-1=3)$ & $5(5-1=4)$ & $4(4-1=3)$ \\
Pergunta 6 & $2(2-5=3)$ & $1(1-5=4)$ & $1(1-5=4)$ & $3(3-5=2)$ & $1(1-5=4)$ \\
Pergunta 7 & $5(5-1=4)$ & $4(4-1=3)$ & $5(5-1=4)$ & $5(5-1=4)$ & $5(5-1=4)$ \\
Pergunta 8 & $2(2-5=3)$ & $1(1-5=4)$ & $1(1-5=4)$ & $1(1-5=4)$ & $1(1-5=4)$ \\
Pergunta 9 & $5(5-1=4)$ & $5(5-1=4)$ & $5(5-1=4)$ & $4(4-1=3)$ & $5(5-1=4)$ \\
Pergunta 10 & $2(2-5=3)$ & $2(2-5=3)$ & $2(2-5=3)$ & $1(1-5=4)$ & $1(1-5=4)$ \\
& $37 \times 2.5=92.5$ & $36 \times 2.5=90$ & $37 \times 2.5=92.5$ & $35 \times 2.5=87.5$ & $38 \times 2.5=95$ \\
\hline
\end{tabular}

Fonte: Autores (2021). 
Gráfico 2 - Respostas ao questionário aplicado aos médicos participantes do teste piloto do aplicativo IntegRAS.

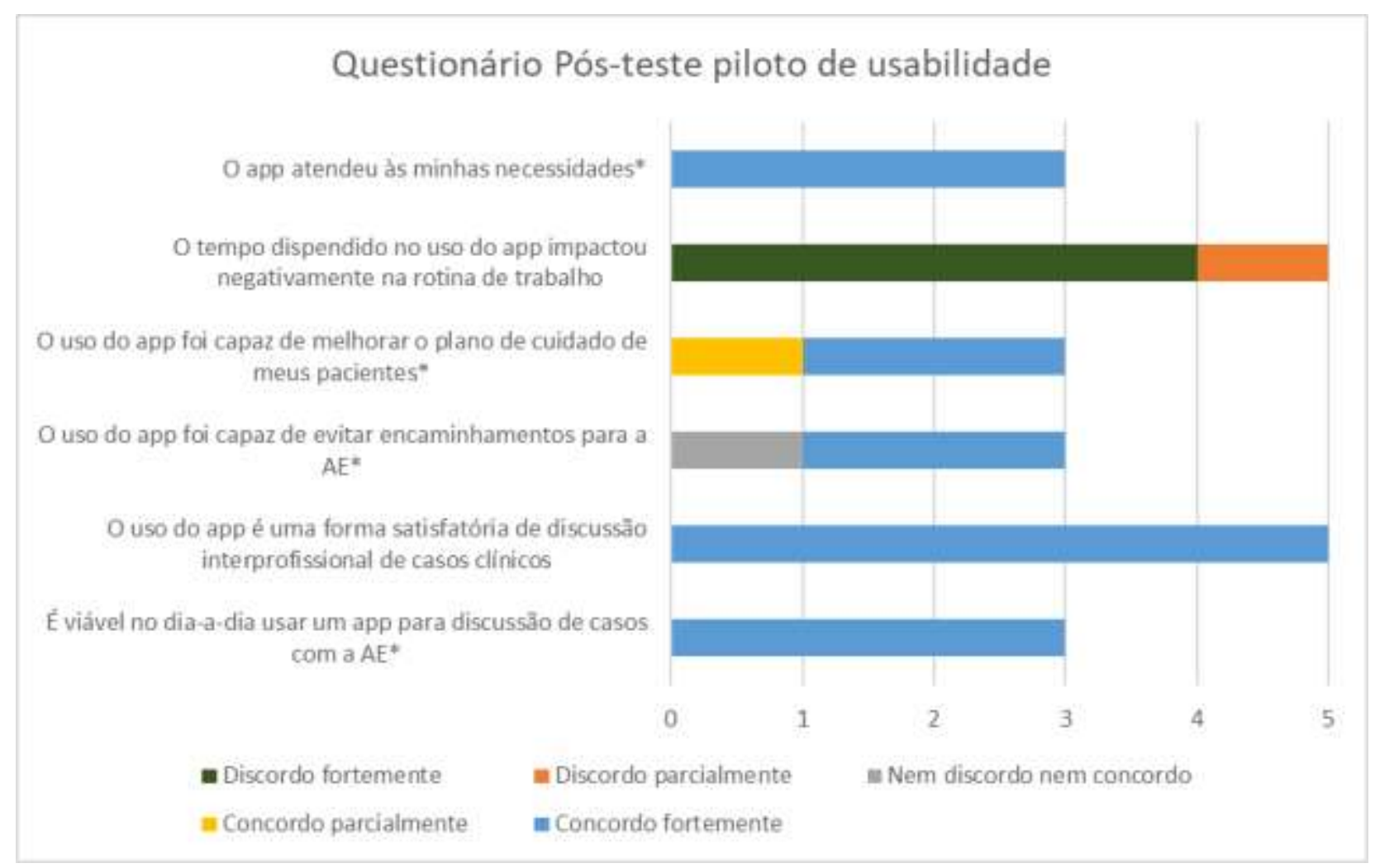

Legenda: * Perguntas restritas apenas aos participantes generalistas (n=3). Fonte: Autores (2021).

\section{Discussão}

$\mathrm{O}$ aplicativo IntegRAS, desenvolvido e testado no presente estudo, foi concebido para facilitar as rotinas de atendimento ambulatorial de profissionais médicos generalistas quando da identificação de questões de manejo clínico que eventualmente estejam fora de seu escopo de atuação. Ao viabilizar um canal de comunicação direta entre estes profissionais e o especialista focal em Endocrinologia, o aplicativo atuou como uma ferramenta de suporte à tomada de decisão clínica, ao mesmo tempo em que se constituiu um instrumento de educação permanente em saúde para os profissionais generalistas da APS.

A utilização das modernas tecnologias em plataformas de comunicação para proporcionar troca de informações e aconselhamento clínico entre profissionais da atenção primária e especialistas é conhecida na literatura pelo termo Electronic Consultation. Tem sido uma estratégia em franca expansão em todo o mundo, trazendo repercussões positivas sobre a regulação dos fluxos de encaminhamento para a $\mathrm{AE}$, com redução da demanda e tempo de espera para consultas presenciais com especialistas, bem como redução de custos envolvidos tanto para os profissionais e instituições de saúde, quanto para os pacientes (Liddy et al., 2016; Muniz et al., 2021; Ray \& Kahn, 2020; Tuot et al., 2018). Alinhado ao conceito e à ampla difusão da Electronic Consultation, e projetado sob a ótica da conjuntura local, o aplicativo IntegRAS atende a demandas específicas do cenário onde foi desenvolvido.

O valor educacional deste tipo de estratégia é inequívoco, com altos níveis de satisfação geral e amplo potencial agregador entre as partes envolvidas. $\mathrm{O}$ apoio à gestão terapêutica e diagnóstica prestado por tais plataformas relaciona-se diretamente a uma maior eficiência e maior produtividade no ambiente da APS (Joschko et al., 2018; Muniz et al., 2021; Tuot et al., 2018).

No que diz respeito à caracterização dos participantes do teste piloto de uso do aplicativo, chama a atenção o fato de que, a despeito da amostra ser composta, em sua maioria, por profissionais com menos de 10 anos de formados, a experiência 
com atendimento por telemedicina foi reportada por apenas dois dos cinco participantes. Este dado pode estar relacionado tanto à baixa utilização deste instrumento como método educativo no contexto da formação médica, quanto à recorrente indisponibilidade de meios digitais para atendimento de pacientes no âmbito do sistema público de saúde brasileiro, cenário de atuação dos médicos integrantes deste estudo.

Fatores apontados como responsáveis pela precariedade deste cenário são a escassez de recursos, instalações físicas defasadas e a insuficiência de equipamentos e materiais de consumo, situação usual de grande parte dos serviços públicos do país e expressão de importante barreira à consolidação de estratégias no campo da telemedicina (Maldonado, Marques, \& Cruz, 2016). Há ampla heterogeneidade quanto à informatização das Unidades Básicas de Saúde (UBS) no Brasil, com percentuais variando de $40 \%$ na região Nordeste, a $89 \%$ na região Sul. Dentre os estados do Nordeste, Sergipe tem o segundo mais baixo índice de informatização das UBS (32\%), atrás apenas do município do Maranhão, com 12\% destas unidades informatizadas (Brasil, 2020).

Quanto à avaliação da usabilidade do aplicativo, diversas estratégias para mensuração de usabilidade de produtos ou serviços podem ser encontradas na literatura (Martins et al., 2015; Pandrini-Andrade et al., 2019). A escolha pela escala SUS se deu por sua capacidade de avaliar diversos tipos de tecnologia (Martins et al., 2015), além de ser facilmente compreendida e rapidamente aplicada no contexto de um teste piloto, tal como o presente estudo. É um instrumento versátil, cujo uso nos últimos anos tem sido amplamente difundido, contando com referências que auxiliam na interpretação de seu escore, além de ser custo-efetivo por não ter direitos autorais (Bangor et al., 2008).

Em nosso estudo, o teste piloto do app denotou pontuações no $S U S$ variando entre 87.5 e 95 , dado que coloca o aplicativo em uma posição ótima na escala de usabilidade, respaldando-o, com sua interface e características atuais, como um recurso tecnológico eficiente na integração entre as equipes de saúde. Ainda, as respostas às questões remanescentes permitiram inferir que o aplicativo atendeu às necessidades dos participantes, tendo sido útil em aprimorar a condução clínica no ambiente da APS, sem impactar negativamente nas rotinas de trabalho, no que diz respeito à demanda adicional ou interferência no tempo despendido para usá-lo em uma solicitação de interconsulta. Tanto generalistas quanto especialistas foram unânimes em considerar viável a utilização de um instrumento desta natureza para discussão de casos clínicos e compartilhamento de cuidados entre APS e AE.

Tendo se mostrado um potente instrumento de interação entre médicos atuantes nos dois primeiros níveis de atenção à saúde, o aplicativo IntegRAS difere dos anteriormente mencionados Figure 1 e MedShr na medida em que traz consigo a possibilidade de estreitar relações entre pares ao conectar de forma direta generalistas e especialistas que façam parte de uma mesma RAS.

Uma limitação às potencialidades apresentadas pelo aplicativo IntegRAS decorre da necessidade de aceitação e uso pelos profissionais atuantes na APS, uma vez que somente através da adesão destes ao sistema proposto neste estudo e sua efetiva utilização cotidiana será possível atingir os objetivos de aconselhamento oportuno e aprimoramento assistencial ora vislumbrados.

Ainda, o presente estudo teve como limitações um teste piloto com pequeno quantitativo de profissionais participantes e o curto período de tempo em que o mesmo ocorreu. Estudos abordando os impactos da utilização cotidiana do aplicativo IntegRAS em um grupo maior de prestadores de cuidados primários em saúde, durante um período mais longo, poderão mostrar os benefícios e dificuldades do uso do aplicativo na assistência à saúde das comunidades.

\section{Conclusão}

Por tratar-se de uma plataforma de comunicação entre profissionais da atenção primária e especialistas na área de endocrinologia, o aplicativo descrito neste estudo tem o potencial de aumentar a resolutividade no ambiente da APS, 
melhorando a qualidade da assistência em saúde prestada neste primeiro nível de atenção e atuando como instrumento de educação permanente aos médicos generalistas. Assim, sugere-se que este aplicativo seja administrado por Secretarias Municipais de Saúde interessadas, e ofertado às equipes de APS e aos especialistas focais que fazem o corpo clínico de retaguarda da $\mathrm{AE}$.

Ademais, por ter sido desenvolvido a fim de permitir a inclusão posterior de conteúdos vinculados a outras áreas da Medicina, o aplicativo IntegRAS abre um leque de possibilidades de cuidado compartilhado entre APS e AE nas mais diversas condições clínicas que requeiram a análise de especialistas.

\section{Referências}

Bangor, A., Kortum, P. T., \& Miller, J. T. (2008). An Empirical Evaluation of the System Usability Scale. International Journal of Human-Computer Interaction, 24(6), 574-594. https://www.tandfonline.com/doi/abs/10.1080/10447310802205776. 10.1080/10447310802205776

Blom, L., Laflamme, L., \& Mölsted Alvesson, H. (2018). Expectations of medical specialists about image-based teleconsultation - A qualitative study on acute burns in South Africa. PLoS One. 13(3):e0194278. https://pubmed.ncbi.nlm.nih.gov/29543847/. 10.1371/journal.pone.0194278

Brasil. Ministério da Saúde. (2015). Endocrinologia e nefrologia. Protocolos de encaminhamento da atenção básica para a atenção especializada; v. 1. Brasília: Ministério da Saúde. https://bvsms.saude.gov.br/bvs/publicacoes/protocolos_atencao_basica_atencao_especializada_endocrinologia.pdf

Brasil. Ministério da Saúde. (2019). PLANIFICASUS: Workshop 1 - A integração da Atenção Primária e da Atenção Especializada nas Redes de Atenção à Saúde. São Paulo: Hospital Israelita Albert Einstein: Ministério da Saúde. https://atencaobasica.saude.rs.gov.br/upload/arquivos/201908/16114710-guiaworkshop-1.pdf

Brasil. Ministério da Saúde. (2020). Relatório Final do Projeto Piloto Conecte SUS: análise dos avanços obtidos entre outubro/2019 e junho/2020 [recurso eletrônico]. Brasília: Ministério da Saúde. http://bvsms.saude.gov.br/bvs/publicacoes/relatorio_projeto_piloto_conectesus_outubro.pdf

Brooke, J. (1996). SUS: A “quick and dirty” usability scale. London: Taylor \& Francis. https://www.taylorfrancis.com/chapters/edit/10.1201/978149871041135/sus-quick-dirty-usability-scale-john-brooke

Coutinho, G. L. (2014). A Era dos Smartphones: Um Estudo Exploratório sobre o uso dos Smartphones no Brasil (Trabalho de conclusão de curso). Faculdade de Comunicação Social, Universidade de Brasília, Brasília, DF, Brasil. Recuperado de: https://core.ac.uk/download/pdf/196880791.pdf

Gonçalves, M. R., Umpierre, R. N., D’Avila, O. P., Heinzelmann, R., Trevisan, L., \& Harzheim, E. (2017). Desafios da atenção primária à saúde no Brasil. UFRGS. https://www.ufrgs.br/telessauders/documentos/producao_cientifica/DESAFIOS\%20DA\%20ATENÇÃO\%20PRIMÁRIA\%20À\%20SAÚDE.pdf

Hasbún Zegpi, C., Rojas-Lechuga, M. J., Contador-González, J., Curi-Tuma, M., \& Sandoval, M. (2020). Teledermatología asincrónica a través de WhatsApp®: experiencia en la Pontificia Universidad Católica de Chile [Assessment of an asyncronic tele dermatology system for primary care physicians via social networking app WhatsApp]. Revista medica de Chile, 148(9), 1289-1294. https://pubmed.ncbi.nlm.nih.gov/33399704/ .10.4067/S003498872020000901289

Joschko, J.; Keely, E.; Grant, R.; Moroz, I.; Graveline, M.; Drimer, N. \& Liddy, C. (2018). Electronic Consultation Services Worldwide: Environmental Scan. J Med Internet Res, 20(12), e11112. https://pubmed.ncbi.nlm.nih.gov/30578187/ . 10.2196/11112

Liddy, C.; Drosinis, P.; Deri Armstrong, C.; McKellips, F.; Afkham, A. \& Keely, E. (2016). What are the cost savings associated with providing access to specialist care through the Champlain BASE eConsult service? A costing evaluation. BMJ open, 6(6), e010920. https://bmjopen.bmj.com/content/6/6/e010920 . 10.1136/bmjopen-2015-010920

Maldonado, J. M. S. V., Marques, A. B., \& Cruz, A. (2016). Telemedicine: challenges to dissemination in Brazil. Cadernos de Saúde Pública 32(2), e00155615. https://www.scielo.br/j/csp/a/54bg8d5mfWmCC9w7M4FKFVq/?lang=pt\# . 10.1590/0102-311X00155615.

Martinez, R., Rogers, A. D., Numanoglu, A., \& Rode, H. (2018). The value of WhatsApp communication in paediatric burn care. Burns: journal of the International Society for Burn Injuries. 44(4), 947-955. https://pubmed.ncbi.nlm.nih.gov/29395403/ . 10.1016/j.burns.2017.11.005

Martins, A. I., Rosa, A. F., Queirós, A., Silva, A., \& Rocha, N. P. (2015). European Portuguese validation of the System Usability Scale (SUS). Procedia Comput Sci. 67:293-300. https://www.sciencedirect.com/science/article/pii/S1877050915031191 . 10.1016/j.procs.2015.09.273

Mendes, E. V. (2011). As redes de atenção à saúde. Brasília: Organização Pan-Americana da Saúde. https://www.paho.org/bra/index.php?option=com_docman\&view=download\&category_slug=servicos-saude-095\&alias=1402-as-redes-atencao-a-saude-2aedicao-2\&Itemid=965

Muniz, M. C. H., Santos, M. A., Araújo, B. C. L., Oliveira, A. A., \& Simões, S. M. (2021). Impacts of electronic communication systems between primary and specialized care: an integrative review. Research, Society and Development, [S. l.]. 10(10), e186101018805. https://rsdjournal.org/index.php/rsd/article/view/18805 . 10.33448/rsd-v10i10.18805 
Padrini-Andrade, L. et al. (2019). Evaluation of usability of a neonatal health information system according to the user's perception. Revista Paulista de Pediatria [online], 37(1), 90-96. https://www.scielo.br/j/rpp/a/T5sJ3dTFcZJrxLhRv9XBQhM/?lang=pt\# . 10.1590/1984-0462

Pecina, J. L., Wyatt, K. D., Comfere, N. I., Bernard, M. E., \& North, F. (2017). Uses of Mobile Device Digital Photography of Dermatologic Conditions in Primary Care. JMIR mHealth and uHealth, 5(11), e165. https://pubmed.ncbi.nlm.nih.gov/29117934/ . 10.2196/mhealth.8257

Peiter, C. C., Santos, J. L. G., Lanzoni, G. M. M., Mello, A. L. S. F., Costa, M. F. B. N. A., \& Andrade, S. R. (2019). Healthcare networks: trends of knowledge development in Brazil. Esc. Anna Nery 23(1). https://www.scielo.br/j/ean/a/8V3GKbxjSp3VdpbR3s78HDb/?lang=pt . 10.1590/2177-9465-EAN2018-0214

Ray, K. N. \& Kahn, J. M. (2020). Connected Subspecialty Care: Applying Telehealth Strategies to Specific Referral Barriers. Acad Pediatr, 20(1), 16-22. https://pubmed.ncbi.nlm.nih.gov/31404707/ . 10.1016/j.acap.2019.08.002

Rizvi, S., Schopf, T., Sangha, A., Ulvin, K., \& Gjersvik, P. (2020). Teledermatology in Norway using a mobile phone app. PloS one, 15(4), e0232131. https://pubmed.ncbi.nlm.nih.gov/32339203/ . 10.1371/journal.pone.0232131

Tuot, D. S.; Liddy, C.; Vimalananda, V. G.; Pecina, J.; Murphy, E. J.; Keely, E.; Simon, S. R.; North, F.; Orlander, J. D. \& Chen, A. H. (2018). Evaluating diverse electronic consultation programs with a common framework. BMC health services research, $18(1)$, 814. https://bmchealthservres.biomedcentral.com/articles/10.1186/s12913-018-3626-4 . 10.1186/s12913-018-3626-4

Wallis, L. A., Fleming, J., Hasselberg, M., Laflamme, L., \& Lundin, J. (2016). A Smartphone App and Cloud-Based Consultation System for Burn Injury Emergency Care. PloS one, 11(2), e0147253.: https://pubmed.ncbi.nlm.nih.gov/26918631/ . 10.1371/journal.pone.0147253

Wiktor, A. J., Madsen, L., Carmichael, H., Smith, T., Zanyk, S., Amani, H., \& Wagner, A. L. (2018). Multiregional Utilization of a Mobile Device App for Triage and Transfer of Burn Patients. Journal of burn care \& research: official publication of the American Burn Association, 39(6), 858-862. https://pubmed.ncbi.nlm.nih.gov/30107518/ . 10.1093/jbcr/iry04

Yang, X., Barbieri, J. S., \& Kovarik, C. L. (2019). Cost analysis of a store-and-forward teledermatology consult system in Philadelphia. J Am Acad Dermatol, 81(3):758-764. https://pubmed.ncbi.nlm.nih.gov/30287316/ . 10.1016/j.jaad.2018.09.036 